\title{
Article \\ PRC1 Stabilizes Cardiac Contraction by Regulating Cardiac Sarcomere Assembly and Cardiac Conduction System Construction
}

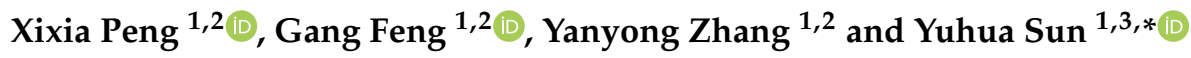 \\ 1 Institute of Hydrobiology, Chinese Academy of Sciences, Wuhan 430072, China; \\ pengxixia1990@163.com (X.P.); fenggangscu@foxmail.com (G.F.); zhangyanyong0924@163.com (Y.Z.) \\ 2 College of Advanced Agricultural Sciences, University of Chinese Academy of Sciences, Beijing 100049, China \\ 3 The Innovation of Seed Design, Chinese Academy of Sciences, Wuhan 430072, China \\ * Correspondence: sunyh@ihb.ac.cn
}

check for

updates

Citation: Peng, X.; Feng, G.; Zhang, Y.; Sun, Y. PRC1 Stabilizes Cardiac Contraction by Regulating Cardiac Sarcomere Assembly and Cardiac Conduction System Construction. Int J. Mol. Sci. 2021, 22, 11368. https:// doi.org/10.3390/ijms222111368

Academic Editors: Mohsin Khan, Tamer Mohamed and Ronald Vagnozzi

Received: 16 September 2021 Accepted: 17 October 2021 Published: 21 October 2021

Publisher's Note: MDPI stays neutral with regard to jurisdictional claims in published maps and institutional affiliations.

Copyright: (c) 2021 by the authors. Licensee MDPI, Basel, Switzerland. This article is an open access article distributed under the terms and conditions of the Creative Commons Attribution (CC BY) license (https:/ / creativecommons.org/licenses/by/ $4.0 /)$.

\begin{abstract}
Cardiac development is a complex process that is strictly controlled by various factors, including PcG protein complexes. Several studies have reported the critical role of PRC2 in cardiogenesis. However, little is known about the regulation mechanism of PRC1 in embryonic heart development. To gain more insight into the mechanistic role of PRC1 in cardiogenesis, we generated a PRC1 loss-of-function zebrafish line by using the CRISPR/Cas9 system targeting $r n f 2$, a gene encoding the core subunit shared by all PRC1 subfamilies. Our results revealed that Rnf2 is not involved in cardiomyocyte differentiation and heart tube formation, but that it is crucial to maintaining regular cardiac contraction. Further analysis suggested that Rnf2 loss-of-function disrupted cardiac sarcomere assembly through the ectopic activation of non-cardiac sarcomere genes in the developing heart. Meanwhile, Rnf2 deficiency disrupts the construction of the atrioventricular canal and the sinoatrial node by modulating the expression of $b m p 4$ and other atrioventricular canal marker genes, leading to an impaired cardiac conduction system. The disorganized cardiac sarcomere and defective cardiac conduction system together contribute to defective cardiac contraction. Our results emphasize the critical role of PRC1 in the cardiac development.
\end{abstract}

Keywords: Rnf2; PRC1; cardiac contraction; sarcomere assembly; cardiac conduction system

\section{Introduction}

As the first organ to generate and function in the embryo, heart development is strictly regulated by various molecules and signal pathways. To become mature and functional, the embryonic heart undergoes a series of complex processes, including the specification and differentiation of cardiac lineages, the morphogenesis of the heart tube, looping, the assembly of cardiac chambers, the constriction of the atrioventricular canal, and the establishment of proper cardiac functions [1-3]. Errors in any of these processes can cause congenital heart malformations $[4,5]$.

The main function of the heart is to drive blood circulation with sturdy contractions triggered by electrical impulses. Defects in cardiac function are often due to abnormalities in the cardiac contractile apparatus or the cardiac conduction system (CCS) [3]. The cardiac contractile apparatus, namely the cardiac sarcomere, provides a structural basis for cardiac contraction [6]. The cardiac sarcomere is mainly composed of two filamentary systems, the thick and thin filaments. The myosin-based thick filaments constitute the electron-dense Abands, whereas the actin-based thin filaments make up the least electron-dense I-bands. The M-lines, anchoring the bipolar thick filaments, are located in the middle of the A-bands. The Z-disc, located in the middle of the I-bands and anchoring the end of the thin filaments and the third filament system in the sarcomere-titin filaments, defines the boundary between two adjacent sarcomere units [6-8]. The development of a functional cardiac sarcomere 
requires promoting the expression of cardiac sarcomere genes and repressing the expression of non-cardiac sarcomere genes in the heart. The ectopic activation of non-cardiac myofibril genes in the developing heart can disrupt cardiac sarcomere formation and cause impaired cardiac function [9].

The CCS is responsible for initiating and propagating proper electrical impulses to generate and maintain coordinated cardiac contraction [10]. The sinoatrial node (SAN) and the atrioventricular canal (AVC) are two critical components of the CCS [10]. The specialized pacemaker myocyte in SAN initiates the electrical impulse. The AVC, where the atrioventricular node and the atrioventricular bundle are located, is critical for the conduction delay and electrical impulse propagation that lead to the coordinated contraction of the atrium and ventricle [10]. The specification of the AVC can be defined by the restricted expression of notch receptor $1 b$ (notch1b), hyaluronan synthase 2 (has2), and activated leukocyte cell adhesion molecule a (alcama) in the endocardium, as well as T-box transcription factor $2 b$ (tbx2b), bone morphogenetic protein 4 (bmp 4$)$, and versican a (vcana) in the myocardium $[3,11]$. Multiple AVC marker genes present a specific change in the expression region from throughout the anteroposterior extent of the heart to specific cells within the AVC, such as bmp4, vcana, and notch1b [11,12]. Expanded expression of AVC marker genes can cause AVC malformation and result in a dysmorphic heart with disordered cardiac contraction $[13,14]$. The gene bmp4 is also expressed in pacemaking cells in the SAN, targeted directly by the homeodomain transcription factor Shox2, which is essential for sinoatrial valve formation and pacemaking system development $[15,16]$.

As well-known epigenetic regulators [17], the Polycomb Group (PcG) proteins are involved in a plethora of biological processes, including epigenetic inheritance [18], Xchromosome inactivation [19,20], stem cell self-renewal [21], senescence [22], and tumorigenesis [23-25]. The PcG proteins primarily form two principal complexes, Polycombrepressive complex 1 (PRC1) and Polycomb-repressive complex 2 (PRC2). PRC2 is responsible for the trimethylation of Lys27 on histone $\mathrm{H} 3$ (H3K27me3) via the EZH2 or EZH1 protein subunit, while PRC1 catalyzes the ubiquitination of Lys119 on histone H2A (H2AK119ub) through E3 ligase RING1 [26,27]. Although PRC1 complexes show high complexity in their composition, RING1 is shared by all PRC1 subfamilies [28]. Two RING1 homologues (Ring1a and Ring1b/Rnf2) located in the mice genomes, and both of them can assemble into PRC1 [28]. Ablating RING1A and RNF2 simultaneously lead to lethal aplasia in mice [26]. Only one RING1 ortholog, namely $r n f 2$, exists in the zebrafish genomes. The $r n f 2$ mRNA distribution was not spatially restricted during development, with obvious expression in the brain and pectoral fin [29]. The deletion of Rnf2 induces severe developmental defects in zebrafish embryos, including craniofacial abnormality and the absence of pectoral fins [29,30]. A recent study has shown that Rnf2 may regulate cardiac looping by repressing tbx2/3 expression [31]. However, the conclusion was mainly drawn from 3 dpf embryos. The mechanism through which Rnf2 regulates early cardiogenesis remains elusive.

To obtain further insight into the role of PRC1 in early cardiogenesis, we generated zebrafish Rnf2 mutants by using the CRISPR/Cas9 system. We found that Rnf2 is crucial to maintaining regular cardiac contraction. Rnf2 loss-of-function disrupts cardiac sarcomere assembly and CCS construction by dysregulating specific transcription programs. Our results emphasized the critical role of PRC1 in the development of zebrafish embryonic hearts.

\section{Results}

\subsection{Loss of Rnf2 Caused Embryonic Lethality with Severe Cardiac Defects}

To investigate the role of PRC1 in embryonic development, we generated an Rnf2 mutant zebrafish line using the CRISPR/Cas 9 system. The exon 3 of $r n f 2$ was targeted and two mutant alleles $\left(r n f 2^{\mathrm{f} 5}\right.$ and $\left.r n f 2^{\mathrm{f} 8}\right)$ were generated, as revealed by genotyping (Figure 1A,B). The two mutant alleles were predicted to encode the truncated proteins lacking both the RING and RAWUL domains (Figure 1C). No obvious difference was found 
between the control and mutant embryos at $24 \mathrm{hpf}$, suggesting that Rnf2 is not required for the formation of a basic body structure in zebrafish. However, $r n f 2^{-1-}$ embryos can be separated from their $\mathrm{rff}^{+/+}$and $\mathrm{rnf}^{+/-}$siblings morphologically as early as $36 \mathrm{hpf}$, as the mobility of $r n f 2^{-/-}$embryos is reduced [29]. At $72 \mathrm{hpf}, r n f 2^{-/-}$embryos exhibited pleiotropic phenotypes, including defective craniofacial structures, small eyes, missing pectoral fins and clear pericardial edema encompassing a stringy heart with weak contractility (Figures 1D,E and S1), which was consistent with previous reports [29-31]. The $r n f 2^{-/-}$ larvae usually die within a week, most likely of severe heart failure.

To gain a more detailed view of the cardiac defects in $r n f 2^{-/-}$embryos, we performed histological analyses on the hearts of $4 \mathrm{dpf}$ embryos. The results showed that the heart in the rnf2 $2^{-1-}$ embryos was spindly, with fusiform atriums and ventricles. Unlike the heart of the wild-type embryos, which displayed a constrictive AVC between the atrial and ventricular chambers and protruded well-formed primitive valve leaflets, the AVCs of the $r n f 2^{-/}$embryos were less constricted and the primitive valve leaflets were missing (Figure 1F). These results suggested that the formation of the AVC and primitive valves are severely disrupted in $r n f 2^{-/-}$embryos.

To verify that the cardiac defects resulted from the functional deficiency of Rnf2, we first collected the assumptive $r n f 2^{-/}$embryos with pericardial edema and stringy hearts and investigated their Rnf2 protein levels through western blotting. As we expected, unlike the wild-type embryos showing high Rnf2 levels, no Rnf2 was detected in the assumptive $r n f 2^{-/}$embryos, and H2AK119ub1 enrichment was also decreased to an almost undetectable level (Figure 1G). The residual H2AK119ubi might have been contributed by maternal Rnf2. We also performed rescue assays by microinjecting exogenous $r n f 2$ mRNA into the 1-cell-stage embryos from $\mathrm{rnf}^{+/-}$self-cross and counted the embryos with and without pericardial edema at $72 \mathrm{hpf}$. The results showed that the overexpression of rnf 2 reduced the percentage of embryos with edema from $32 \%$ to $17 \%$ (Figure $1 \mathrm{H}$ ). As expected, the percentage of embryos with the stringy heart phenotype was also reduced to a similar extent. These data strongly suggested that the observed heart defects were caused specifically by Rnf2 loss-of-function.

\subsection{The Cardiac Contraction Was Disrupted in the Rnf2-Null Embryos}

It was observed that the hearts of the $r n f 2^{-1-}$ embryos contracted much slower than those of the wild-type at $72 \mathrm{hpf}$. To determine when the cardiac contractions began to be affected, we measured the heart rates of the $r n f 2^{-/-}$and wild-type embryos at different developmental stages. In the wild-type embryos, the heart started to contract by about $20 \mathrm{hpf}$ and sped up with development (Figure 2). The hearts of the $r n f 2^{-/-}$embryos started to contract by about $20 \mathrm{hpf}$ as well, but the heart rates increased more slowly, leading to a reduced heart rate at $24 \mathrm{hpf}$ (Figure 2). Furthermore, the heart rates of the $r n f 2^{-/-}$embryos even decreased at $84 \mathrm{hpf}$ (Figure 2). These data showed that the cardiac contraction of $r n f 2^{-/-}$embryos was affected as early as $24 \mathrm{hpf}$, indicating that defects in cardiac development began no later than $24 \mathrm{hpf}$. 
A

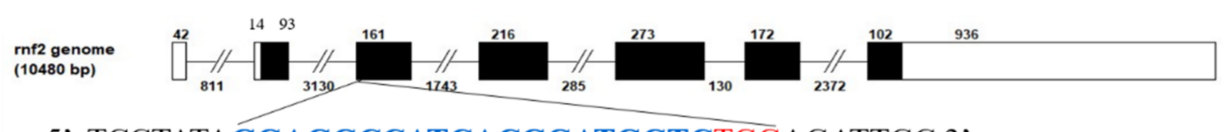

5' TCCTATAGGAGGCCATCACGGATGGTCTGGAGATTGC 3'

B

WT 5' TCCTATAGGAGGCCATCACGGATGGTCTGGAGATTGC 3'

$r n f^{f 5} 5$, TCCTATAGGAGGCCATCACGGATGG -------- AGATTGC 3' (-5bp)

$r n f^{f s} 5^{\prime}$ TCCTATAGGAGGCCATCACGGATG --------- ATTGC 3' (-8bp)

T CCTATAGG A G GC CAT CACG G AT G G ${ }^{\downarrow}$ A G AT T GCA GT G T C C

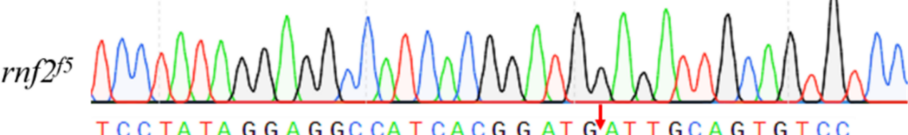

TCC TATAGGAGGC CA T CACG G AT GAT T GCAGTGTCC

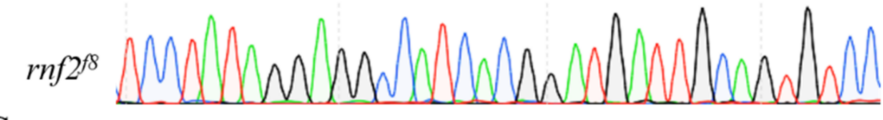

C

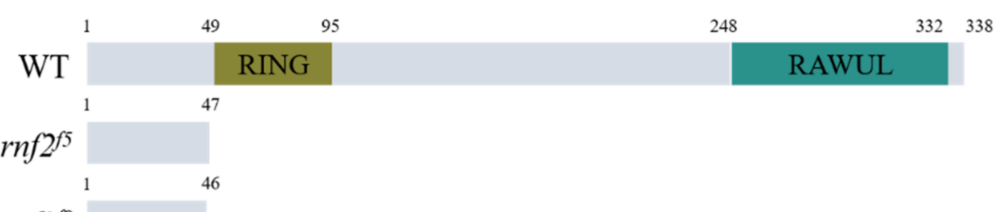

$r n f 2^{f 8}$

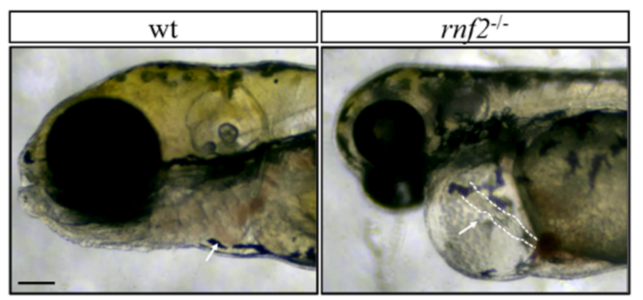

E

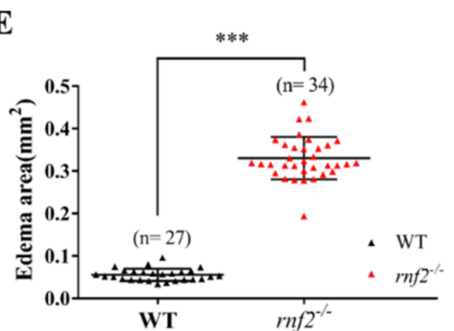

G
F
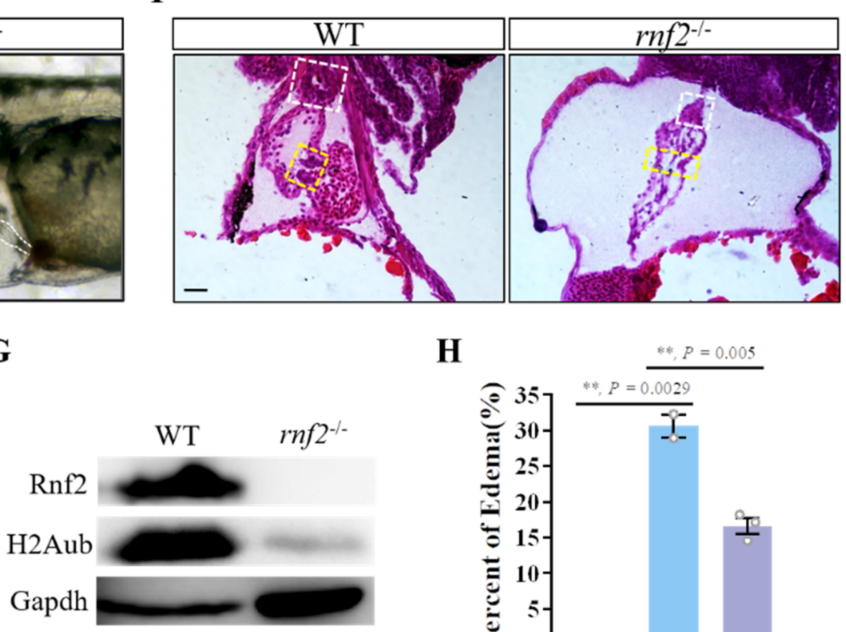

H

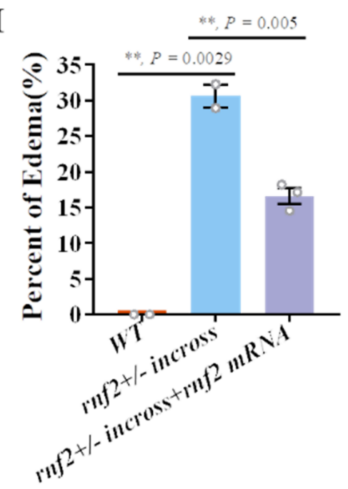

Figure 1. Rnf2-null zebrafish mutant displayed severe cardiac defects. (A) The target site (sequence highlighted in blue) is located in exon 3. The PAM site is underlined and highlighted in red; (B) DNA sequencing identified that two mutant alleles carried a $5 \mathrm{bp}\left(r n f 2^{\mathrm{f} 5}\right)$ and $8 \mathrm{bp}\left(r n f 2^{\mathrm{f} 8}\right)$ deletion respectively. The deletion sites are indicated by red arrows; (C) Schematic diagram of the wild-type and mutant Rnf2 proteins. Zebrafish wild-type Rnf2 contained an N-terminal Ring-finger domain (yellow) and a C-terminal RAWUL domain. The two mutant proteins were truncated before the RING-finger domain; (D) The $r n f 2^{-/-}$larvae displayed severe pericardia edema. The hearts are indicated by white arrows, the stringy heart in $r n f 2^{-/-}$ is depicted by white dotted lines, scale bar: $0.2 \mathrm{~mm}$; (E) Scatter plot showing the sectional area of edema in wild-type (black) and $r n f 2^{-/-}$(red). (F) Histological sections of $4 \mathrm{dpf}$ heart, boxes in yellow indicate the AVC and valves, white boxes indicate the bulbous arteriosus, scale bar: $50 \mu \mathrm{m}, \mathrm{n}(\mathrm{WT})=2, \mathrm{n}\left(\mathrm{rnf} 2^{-/-}\right)=3$; (G) Western blot verified the deletion of Rnf2 protein. Gapdh was set as internal reference; $(\mathbf{H})$ Bar graph showing the percentage of embryos with edema. ${ }^{* *}, p<0.01$; $* * *, p<0.001 ; \mathrm{n}$, sample number. 


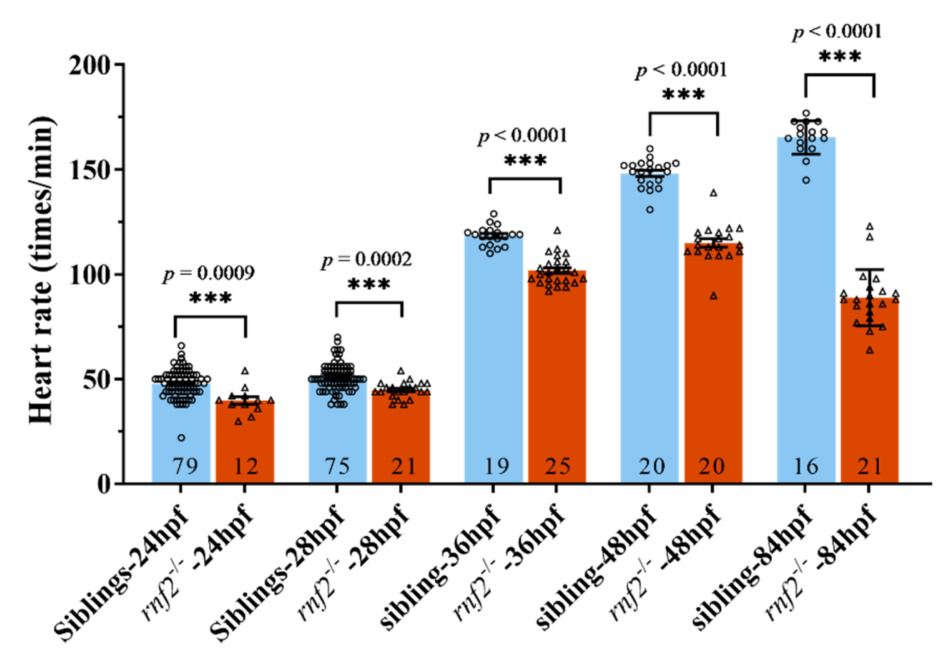

Figure 2. The cardiac contraction was disrupted in Rnf2-null zebrafish embryos. Scatter plot showing the heart rates of wild-type (blue) and $r n f 2^{-/-}$(red) embryos at different developmental stages. Numbers in the bottoms of the bars indicate the sample number of each group. ${ }^{* *}, p<0.001$.

\subsection{The Mesoderm Formed Normally in the Rnf2-Null Zebrafish Embryos}

During the cardiogenesis process, both cardiomyocytes and endocardial cells originate in the mesoderm [32-34]. Since the cardiac contractions of the $r n f 2^{-/-}$embryos were affected just as the hearts started beating, we wondered whether the cardiac development became defective even earlier. To investigate whether the cardiac defects resulted from aberrant mesoderm formation, we examined the expression of the representative mesoderm markers eve1 (ventral mesoderm), flh (axial mesoderm), and foxc1a (paraxial mesoderm) at $8 \mathrm{hpf}$ by using WISH. The WISH results showed that these mesoderm markers expressed normally in the absence of Rnf2 (Figure 3), indicating that mesoderm formation was not affected in the $r n f 2^{-/-}$embryos.

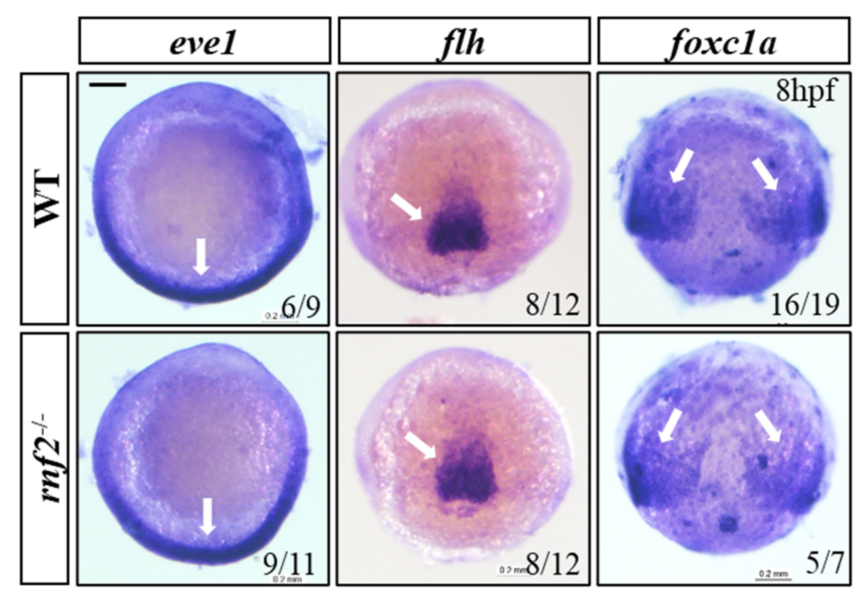

Figure 3. The mesoderm formed normally in Rnf2-null zebrafish embryos. WISH results showing expression of mesoderm markers eve1 (ventral mesoderm), flh (axial mesoderm), and foxc1a (paraxial mesoderm). The expression regions are indicated by white arrows. Scale bar: $0.2 \mathrm{~mm}$.

\subsection{The Heart Tube Structure Appeared Normal in the rnf2 $2^{-1}$ Embryos}

Given the normal mesoderm formation, we wondered whether the cardiac progenitor specification, migration, and heart tube generation were affected in the $r n f 2^{-/-}$embryos. We first examined the expression of pan-myocardial gene cardiac myosin light chain 2 (cmlc2) at different developmental stages. The results showed that at $24 \mathrm{hpf}$ and $36 \mathrm{hpf}$, the expression of $\mathrm{cmlc} 2$ in the $r n f 2^{-1-}$ embryos displayed no obvious difference from that of the wild-types (Figure $4 \mathrm{~A}$ ). At $48 \mathrm{hpf}$ and $72 \mathrm{hpf}$, except for the alteration in the shape 
of the expression region, which may have been due to variations of the heart shape, the transcription level of $\mathrm{cmlc} 2$ still showed no obvious difference (Figure 4A). We then detected the expression of the atrial and ventricular myocardium-specific genes atrial myosin heavy chain (amhc) and ventricular myosin heavy chain (vmhc). The expression of amhc and vmhc showed no significant anomalies (Figure 4B). The expression of the cardiac regulation genes tbx20 and natriuretic peptide A (nppa) was normal as well (Figure 4C,D). These results indicated that the myocardial specification and migration were largely normal in the $r n f 2^{-/-}$embryos.
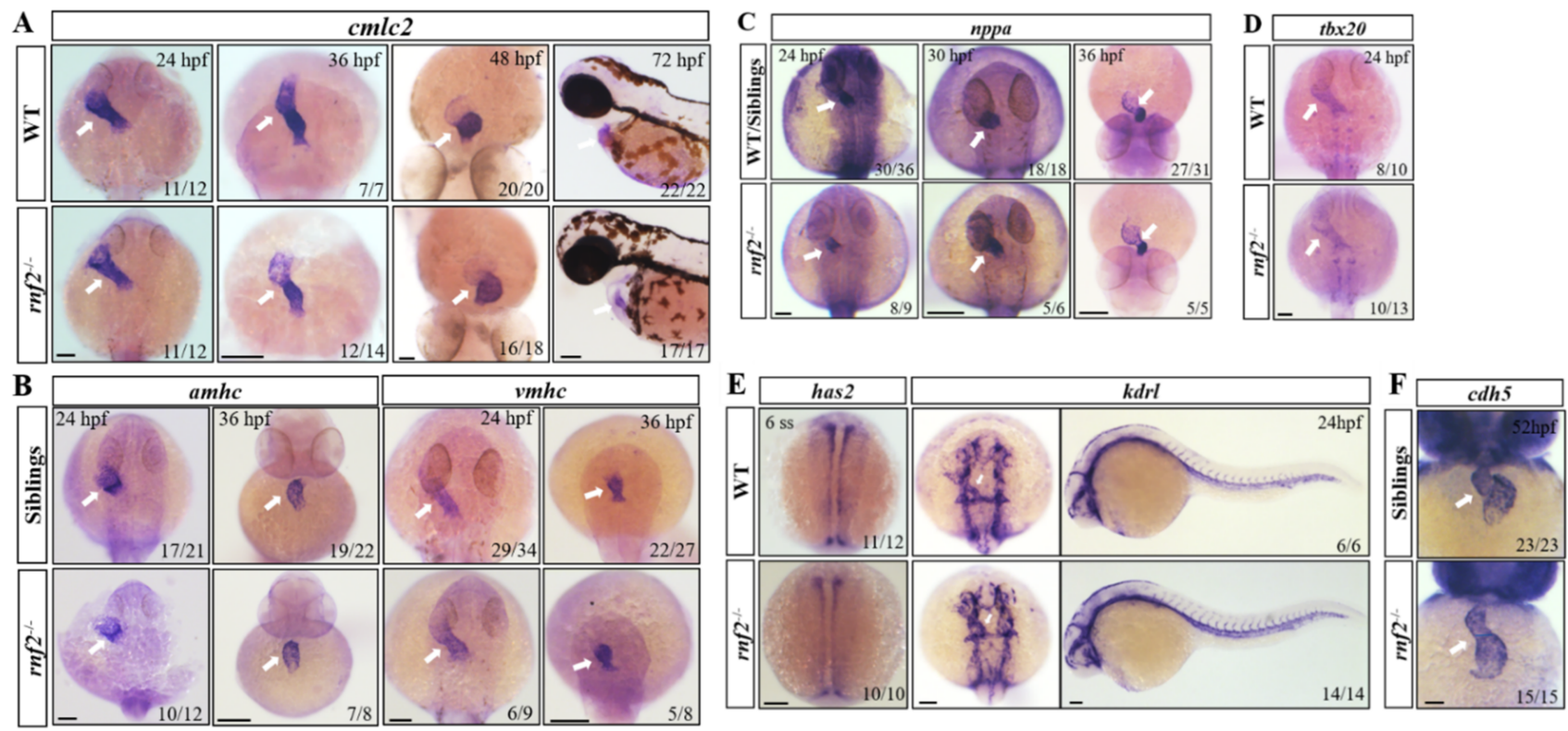

Figure 4. Expression of cardiomyocyte markers examined by in situ hybridization. (A) cmlc2 at different developmental stages; (B) Atrial ( $a m h c$ ) and ventricle ( $v m h c$ ) cardiomyocyte markers at $24 \mathrm{hpf}$ and $36 \mathrm{hpf}$; (C) Growth factor $n p p a$ at $24 \mathrm{hpf}$, $30 \mathrm{hpf}$, and $36 \mathrm{hpf}$; (D) tbx20 at 24 hpf; (E) Expression of endocardial precursor marker gene has2 and endothelial marker gene $k d r l$ in the heart and blood vessels, white arrows indicate the heart tube; (F) Expression of endothelial marker gene $c d h 5$ in the heart. The expression of genes in the heart is indicated by white arrows. Scale bar: $0.2 \mathrm{~mm}$.

The heart tube is composed of an outer myocardial layer and an inner endothelial layer at early stages. We also examined the expression of the endothelial marker has 2 at 6-somite stage, as well as kinase insert domain receptor-like ( $k d r l)$ at $24 \mathrm{hpf}$ and cadherin 5 (cdh5) at $52 \mathrm{hpf}$. The results showed the normal expression of all these three genes, indicating that the endocardium largely formed normally in the $r n f 2^{-/-}$embryos (Figure 4E,F). These results suggested that cardiac progenitor specification and migration are not strictly dependent on Rnf2, and that the heart tube formation appeared normal in the $r n f 2^{-/}$embryos.

\subsection{Rnf2 Deficiency Disorganized the Sarcomere Assembly in Zebrafish Hearts}

Cardiac contraction deficiency is often due to abnormalities in the cardiac contractile apparatus or the CCS [3]. The cardiac sarcomere is the contractile unit of the heart, providing a structural basis for cardiac contraction [6]. To verify whether the cardiac sarcomere assembly was disrupted in the $r n f 2^{-/-}$embryos, we first detected the transcription of genes associated with non-cardiac myofiber, including skeletal and smooth muscle myofibers, in the embryonic hearts. Compared with the controls (wild-type or sibling embryos), the expression of skeletal and smooth muscle genes was dysregulated in the hearts of the $r n f 2^{-/-}$embryos (Figure 5A). At $24 \mathrm{hpf}$, the expression of the skeletal muscle genes acta1a, $m y l 1$, tnni2b, and tnnt3a was significantly upregulated, while the expression of the cardiac sarcomere gene $a c t a 1 b$ and the smooth muscle genes myl6, myl9b, and ppp1r12 was not affected (Figure 5A). Later on, the transcription levels of cardiac acta1b and the smooth muscle genes acta2, myl9b, ppp1r12, and myh11a were elevated gradually and all were up- 
regulated significantly at $48 \mathrm{hpf}$, while the expression of skeletal muscle genes was reduced gradually and downregulated significantly at $48 \mathrm{hpf}$ (Figure 5A). These results prompted us to deduce that the cardiac sarcomere of the $r n f 2^{-/}$embryos may have been defective. To verify this deduction, we further examined the cardiac structure of the wild-type and mutant embryos by using TEM. The results showed that the cardiac sarcomere structure of the $r n f 2^{-/-}$embryos was different from that of the wild-types (Figure 5B). The myofibril fibers in the rnf2 $2^{-/}$embryos were arranged more tightly. The I-band and Z-disc in the $r n f 2^{-/-}$hearts were significantly wider than in those of the wild-type embryos (Figure 5C). Thus, we propose that the loss of Rnf2 may trigger defective cardiac sarcomere alignment via the ectopic transcription activation of non-cardiac sarcomere genes in the developing heart, contributing to severe cardiac contractile defects in $r n f 2^{-/-}$embryos.

A
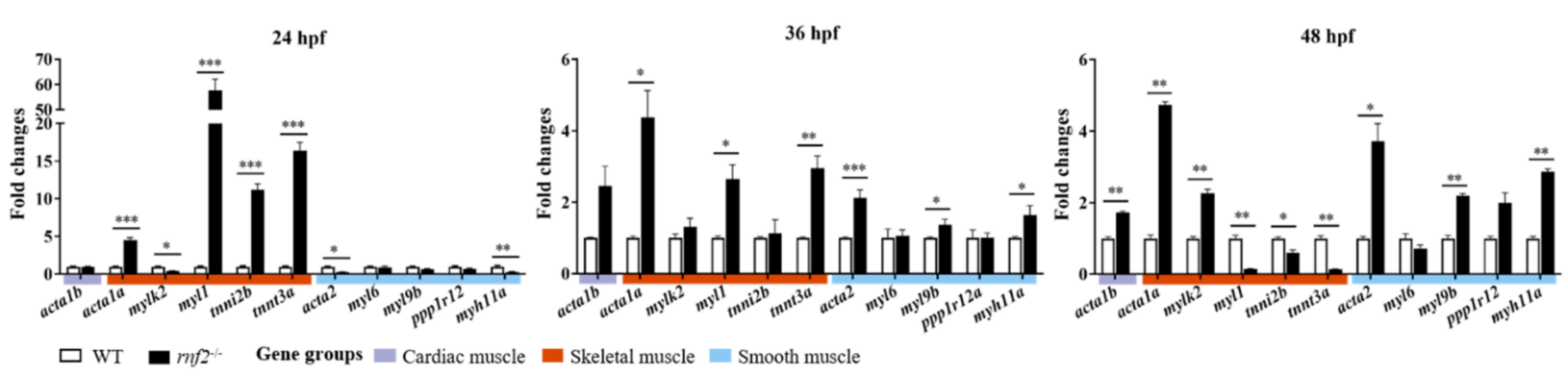

B
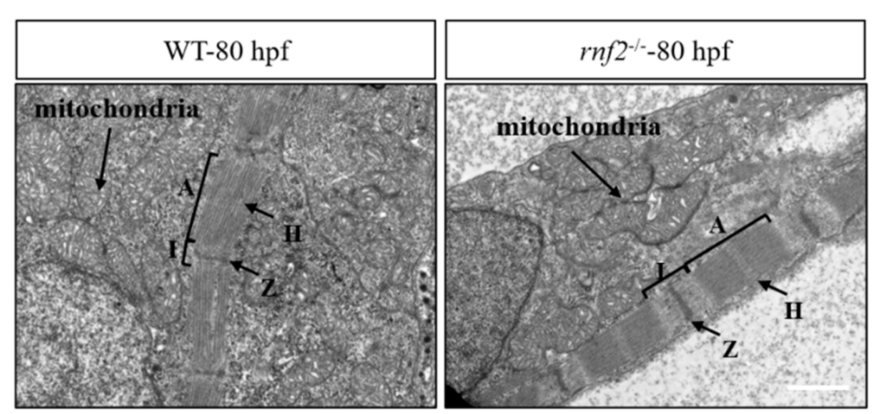

C
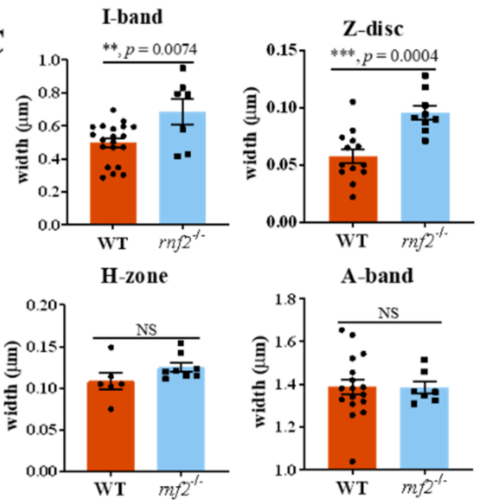

Figure 5. Rnf2 deficiency disorganized sarcomere assembly in zebrafish hearts. (A) Real-time PCR tested the expression of skeletal and smooth muscle genes at $36 \mathrm{hpf}$ (left) and $48 \mathrm{hpf}$ (right). The fold changes of relative mRNA levels are presented as mean \pm SEM. The expression in wild-types was normalized to 1 . The experiment was repeated on three separate occasions. $\mathrm{n}=159,111$, and 161 for $r n f 2^{-/}$group at $24 \mathrm{hpf}, 36 \mathrm{hpf}$, and $48 \mathrm{hpf}$, respectively; $\mathrm{n}=145,113$, and 164 for WT group at $24 \mathrm{hpf}, 36 \mathrm{hpf}$, and $48 \mathrm{hpf}$, respectively. (B) Cardiac TEM revealed the sarcomere of cardiac muscle was abnormal in $r n f 2^{-/-}$hearts. A, A-band; I, I-band; H, H-zone; Z, Z-disc. Scale bar: $1.0 \mu \mathrm{m} . \mathrm{n}=3$. (C) Bar graph showing the width of A-band, I-band, Z-disc, and H-zone in wild-type and $r n f 2^{-/}$cardiac sarcomeres. ${ }^{*}, p<0.05 ;{ }^{* *}, p<0.01$; $* * *, p<0.001 ; \mathrm{NS}$, no significant.

\subsection{Rnf2 Deficiency Caused Defects in the Cardiac Conduction System}

As well as abnormalities in the contractile apparatus, the deficiency in the CCS can cause contraction defects. Histological analysis showed that the AVC in the $r n f 2^{-/-}$ embryos was not as constrictive as in the wild-type embryos. The AVC is a core component of the CCS; this prompted us to speculate that the CCS in the $r n f 2^{-/-}$embryos may be defective. To confirm this speculation, we first examined the AVC patterning by detecting the expression of vcana and alcama in $r n f 2^{-/-}$embryos by using WISH (Figure 6A). In the control embryos, the AVC was well formed between the atrial and ventricular chambers, and the expression of alcama and vcana was restricted to the AVC region. By contrast, the $r n f 2^{-1-}$ embryos lacked a clear AVC ring, and the mRNA distribution of alcama and vcana was diffused and extended into the flanking chambers, instead of being restricted 
to the AVC (Figure 6A). These results indicated that the AVC constriction was disrupted in the hearts of the $r n f 2^{-/}$embryos. Furthermore, we detected the expression of $b m p 4$ at $48 \mathrm{hpf}$ and $56 \mathrm{hpf}$ in the $r n f 2^{-/-}$embryos (Figure 6B). With a shorter staining time of $48 \mathrm{hpf}$ embryos, the expression of bmp 4 at the AVC was expanded in the $r n f 2^{-/-}$embryos, as was that of alcama and vcana (Figure 6B). Furthermore, at $56 \mathrm{hpf}$, with a longer staining time, the expression of bmp 4 at SAN was clearly reduced (Figure 6B), indicating that the specialized pacemaker myocytes in SAN were decreased. These data suggested that the CCS was disrupted in the $r n f 2^{-/}$embryos.
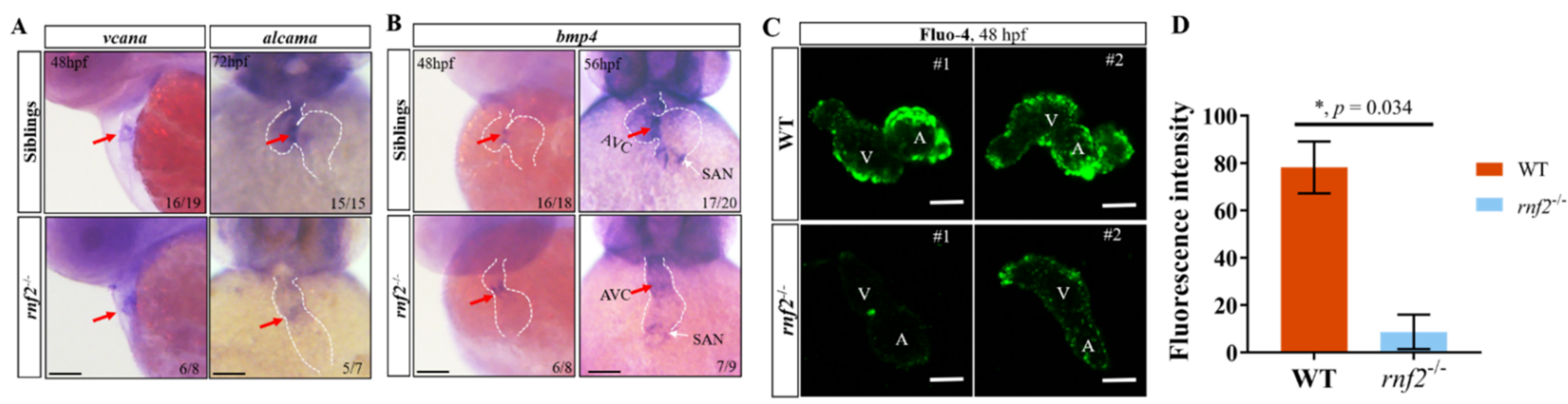

Figure 6. Rnf2 deficiency caused defects in the cardiac conduction system. (A) Expression of AVC myocardial markers vcana and alcama; (B) Expression of AVC endocardial and SAN marker bmp4. Red arrows indicate AVC; (C) Fluo-4 staining detects calcium signal intensity in the hearts of $r n f 2^{-/-}$or wild-type embryos; $\mathrm{V}$, ventricle; $\mathrm{A}$, Atrium; \#1/\#2, sample number; $\mathrm{n}=2$. (D) Quantification of fluorescence intensity in Figure 6C. *, $p<0.05$; Scale bar: $0.2 \mathrm{~mm}$.

Calcium signaling is a crucial indicator of CCS function. To map the cardiac conduction optically, we monitored the calcium signal intensity in individual embryonic hearts using a calcium-sensitive dye, fluo-4 AM [35]. Our results showed that the calcium signal intensity in the hearts of the $r n f 2^{-/-}$embryos was dramatically reduced when compared with that of the wild-types (Figure 6C,D), which further supported the argument that Rnf2 deficiency disrupted the CCS in the embryonic hearts, indicating the crucial role of Rnf2 in zebrafish CCS construction.

Taken together, our results suggested that Rnf2 helps to generate proper cardiac contraction by regulating the expression of genes associated with cardiac sarcomere assembly and the cardiac conduction system.

\section{Discussion}

Cardiac development is a complex process strictly controlled by various factors, including the PcG protein complexes. Cardiac-conditional EZH2 deficiency causes severe cardiac defects, including persistent hypertrabeculation, right ventricular hypoplasia, atrial and ventricular septal defects, myocardial fibrosis, and moderately impaired left ventricular systolic function [36]. Zebrafish ezh2 mutant embryos also displayed cardiac differentiation defects, leading to stringy hearts [37]. Reducing embryonic ectoderm development protein (EED), a crucial component of PRC2, in developing cardiomyocytes leads to lethal cardiogenesis defects [36]. These works indicated the critical role of epigenetic repression established by PRC2 in cardiogenesis. Similarly, PRC1 subunits, such as Ring1b, Bmi1/Pcgf4, and Rae28/Phc1, were also reported as playing crucial roles in cardiac development [38-41]. The simultaneous deletion of RNF2 and RING1A results in the loss of function of the entire PRC1 family. However, the functional ablation of mouse Rnf2 causes gastrulation arrest and results in embryonic lethality [42], which prevented us from investigating the function of PRC1 in cardiogenesis. Rnf2 ${ }^{\mathrm{I53}} \mathrm{A} / \mathrm{I} 33 \mathrm{~A}$ mouse embryos, which ablate the E3 ligase activity but retain the ability to incorporate RNF2 complexes into PRC1, exhibit pericardial edema at E15.5, suggesting defects in the developing cardiovascular system [43,44]. Zebrafish Rnf2 was reported to regulate cardiac looping by repressing the transcription of $t b x$ genes [31]. Through single-heart RNA-seq analysis, Chrispijn et al. 
showed that $t b \times 2 / 3$ were upregulated in the hearts of Rnf2 mutants at $2 \mathrm{dpf}$ and/or $3 \mathrm{dpf}$ (but not $1 \mathrm{dpf}$ ) [31]. However, we found that the heart rates of $r n f 2^{-/-}$embryos were reduced significantly as early as $24 \mathrm{hpf}$, indicating that cardiac contraction was disrupted at no later than $24 \mathrm{hpf}$. Our data suggest that Rnf2 may be involved in different processes of zebrafish cardiogenesis through the epigenetic regulation of different transcriptional programs. This inference is consistent with the consensus repression mechanisms by which PcG complexes control the transcription of target genes through chromatin compaction and genome-wide histone modification [45-47], and is substantiated by our finding that Rnf2 regulates zebrafish cardiac contraction by stabilizing proper transcription programs of sarcomere genes and CCS associated genes in the heart.

1. PRC1 maintains regular cardiac sarcomere assembly by orchestrating the sarcomere gene transcription program.

The patterning of the embryonic heart requires proper cardiomyocyte differentiation and the assembly of specific cell types into a functional apparatus. The cardiac sarcomere is the basic contractile unit of myofibrils in the heart [48], forming the structural foundation of cardiac contraction. Disturbance of the cardiac sarcomere structure disrupts contractile activity and causes heart failure phenotypes $[36,49,50]$. The correct generation of a functional cardiac sarcomere requires the activation of cardiac myofibril structural genes and, simultaneously, repression of the expression of non-cardiac sarcomere paralogs in the developing heart. Such extensive orchestration of transcription coincides with the mechanisms of epigenetic transcription control. Indeed, nucleosome remodeling and the deacetylase (NuRD) and PRC2 complexes were both reported as being involved in this transcriptional control, directly or indirectly $[9,36,50]$. CHD4, the catalytic core of the NuRD complex, regulates skeletal- and smooth-muscle-specific transcription programs by directly binding to their promoters. The cardiac conditional null of CHD4 disrupted the cardiac sarcomere structure by inducing the ectopic expression of non-cardiac sarcomere genes in the developing heart [9]. EZH2, the core catalytic subunit of PRC2, represses the transcription of non-cardiac muscle genes in the developing heart by repressing the expression of Six1, which serves to activate the transcription of skeletal muscle genes [36,50]. However, the role of PRC1, another crucial repressive epigenetic regulator, in this transcription control has not been reported. We propose here that the PRC1 complex also plays a critical role in stabilizing the transcription program of sarcomere genes in the developing heart. A group of non-cardiac muscle genes were misexpressed in the developing hearts of Rnf2 mutants. At $24 \mathrm{hpf}$, the skeletal sarcomere structural genes acta1a, myl1, tnni2b, and tnnt3a were all upregulated. The ectopic activation of skeletal muscle genes in the early heart disrupts the cardiac sarcomere, as revealed by TEM, leading to cardiac contraction deficiency. We conclude that PRC1 regulates cardiac sarcomere assembly by repressing the expression of non-cardiac sarcomere genes in the developing heart.

2. PRC1 represses sarcomere genes indirectly.

In another manuscript, which is currently under preparation, we performed a ChIPseq analysis of Rnf2 in $15 \mathrm{hpf}$ zebrafish embryos. No Rnf2 binding was detected on the promoters of these skeletal muscle genes (data not shown). The same result was shown in the $3 \mathrm{dpf}$ Rnf2 ChIP-seq analysis reported by Chrispijn [31]. These data indicate that PRC1 represses the transcription of non-cardiac sarcomere genes in nascent hearts indirectly. PcG complexes attain transcription repression through histone modifications and chromatin compaction [45-47]. Different chromatin recruitment models of PRC1 and PRC2 have been shown in specific tissues and cells or biological processes. The classic model proposes that the recruitment of PRC1 depends on the prior presence of PRC2. PRC2 is first recruited to specific genomic loci and catalyzes H3K27me3. The H3K27me3 marks are recognized by the CBX component and then PRC1 is recruited via the chromodomain of CBX proteins [46,51,52]. Recently, an alternative recruitment model, proposing that PRC1 is targeted to chromatin without pre-existing PRC2 and H3K27me3, was proposed in X chromosome inactivation and mouse embryonic stem cells $[20,25,53,54]$. 
Conversely, the H2AK119ub deposited by PRC1 facilitates the subsequent recruitment of PRC2 [54]. The recruitment model of PRC complexes in the cardiac sarcomere assembly is still unclear. PRC2/EZH2 was reported to repress the transcription of skeletal muscle genes in developing heart by repressing the expression of Six1 $[36,50]$. We consulted the ChIP-seq data reported by Chrispijn and found low-level Rnf2 binding at the promoter region of six1 [31], suggesting that Rnf2 may also restrain the expression of skeletal muscle genes in the heart by repressing six 1 transcripts. We also found high-level H3K27me3 enrichment at six1 promoters, and the H3K27me3 enrichment was retained with Rnf2 deletion [31]. These indicated that both PRC1 and PRC2 complexes are targeted to the promoter region of six1, whereas the recruitment of PRC2 here is independent of PRC1. However, much more evidence is needed to confirm this speculation and to determine whether the recruitment of PRC1 depends on the pre-recruitment of PRC2, or whether each are targeted to chromatin independently.

3. PRC1 is involved in the construction of the zebrafish cardiac conduction system.

The main function of the CCS is to generate and conduct cardiac impulses to initiate and maintain rhythmic contractions. The impaired formation or malfunction of CCS components, including SAN dysfunction and AV blockage, can cause cardiac conduction disease (CCD), accompanied by severe arrhythmias [55]. The mammal CCS is mainly composed of pacemaker cardiomyocytes in SAN, AVN, and the ventricular conduction system (VCS) [10]. Although the zebrafish CCS features no AVN or VCS, it does feature slow-conducting AVC cardiomyocytes, which act as functional alternatives to mammalian AVN cardiomyocytes $[10,56]$. A better understanding of the development of the CCS's components can help to illuminate the pathological and molecular mechanisms leading to CCD. Although knowledge related to the electrophysiological properties of CCS structures and functions has increased steadily since the anatomy of the CCS was identified 100 years ago, the molecular and genetic foundation of CCS specification and formation remain unclear [57]. Here, we demonstrated that Rnf2/PRC1 plays a critical role in the construction of the zebrafish CCS. The AVC formation was disrupted and the expression of AVC-specific markers, including bmp4, vcana and alcama, was expanded into flanked chambers instead of constricting in the AVC ring with loss of Rnf2 function. This indicates that PRC1 is required for the correct formation of CCS through the restriction of the AVC-specific transcriptional program. The CCS fulfills its function by propagating electrical impulses. The malformation of CCS components can induce changes of electrical signaling [58]. The decreased calcium signaling intensity in the hearts of Rnf2 mutants further confirms our conclusion. Our data provide more information on the genetic regulation of CCS construction and may facilitate the development of new treatment strategies for CCD.

Collectively, we generated a PRC1-null zebrafish line by knocking out the core PRC1 subunit, Rnf2. Our data indicate that PRC1 modulates cardiac sarcomere assembly by stabilizing sarcomere gene transcription programs, and regulates the specification of basic structures in the CCS by regulating the expression of AVC- and SAN-specific genes in the developing heart, which helps to maintain regular cardiac contraction. It is the first time that PRC1 has been reported to function in cardiac sarcomere assembly and cardiac conduction system construction during embryonic heart development. Our data offer a more detailed image of the function of PRC1 in vertebrate cardiac development. This may provide some new insights into the pathology of cardiac diseases caused by PRC1 loss-of-function.

\section{Materials and Methods}

\subsection{Zebrafish Lines and Maintenance}

$A B$ line zebrafish were used in this study. The zebrafish were raised and maintained according to standard laboratory procedures as stated in the zebrafish book [59]. 


\subsection{Generation of Rnf2 Mutants}

The zebrafish Rnf2 mutants were generated using the CRISPR/Cas9 system. The guide RNA (gRNA) was designed by targeting the exon 3 of the rnf2 gene. Embryos at the 1cell stage were co-injected with $200 \mathrm{ng} / \mu \mathrm{L}$ Cas 9 mRNA and $80 \mathrm{ng} / \mu \mathrm{L}$ gRNA. The genomic DNA of 20 injected embryos at $24 \mathrm{hpf}$ was extracted and subjected to PCR amplification. A DNA fragment containing the rnf2 target site was amplified by PCR using the primers $5^{\prime}$-TTGAGGTAGTTGCTCCCAAAG-3' and 5'-GGCATTCCTTGGTGGTCATA- ${ }^{\prime}$, and the genotype was confirmed by DNA sequencing.

\subsection{Western Blotting Experiments}

The Western blot analysis was performed as previously described [60]. About $50 \mathrm{em}-$ bryos were collected and dechorionated, and cell lysates were prepared using a TNE buffer (50 mM Tris- $\mathrm{HCl}$ ( $\mathrm{pH} 8.0), 150 \mathrm{mM} \mathrm{NaCl}, 1 \%$ Triton X-100, 0.5\% sodium deoxycholate, $5 \mathrm{mM}$ EDTA, $1 \times$ DTT) with the cOmplete proteinase inhibitor (Roche, Switzerland). The lysates were resolved on 15\% SDS polyacrylamide gels and immunoblotted with primary antibody anti-Rnf2 (A302-869A, Bethyl). The images were captured with an Image Quant LAS 4000mini (GE Healthcare Life Sciences, Chicago, IL, USA).

\subsection{Whole Mount In Situ Hybridization (WISH)}

The WISH was performed as previously described [61]. The DIG-labeled anti-sense probes were generated using a DIG RNA Labeling Kit (SP6/T7) (Roche, Switzerland). The DNA templates used to generate the probes were amplified by PCR and the primers used are listed in Supplementary Table S1. For embryos at or after $36 \mathrm{hpf}$, the homozygotes were separated from their siblings according to their mobility and pectoral fin phenotype. For embryos before $36 \mathrm{hpf}$, as it was difficult to separate homozygous mutants from the heterozygous and wild-type siblings, WISH was performed for all self-cross progenies of $r n f 2^{+-}$parents. After the WISH, each embryo was photographed and genotyped separately. The photographs were taken under a stereomicroscope (Leica Z16 APO) with a digital camera (Leica DFC450).

\subsection{Histological Analysis}

The zebrafish embryos at $4 \mathrm{dpf}$ were fixed with Bouin's solution at room temperature for $24 \mathrm{~h}$. The fixed embryos were embedded in 1\% agarose, dehydrated with gradient ethanol, hyalinized with xylene, and then embedded in paraffin. The paraffin-embedded embryos were sectioned, and the sections were stained with standard hematoxylin and eosin staining.

\subsection{Quantitative Real-Time PCR}

The relative mRNA levels of the genes in the $r n f 2^{-/-}$and control hearts were detected by using quantitative real-time PCR (qRT-PCR). The primers used are listed in Supplementary Table S2. Individual hearts from embryos were isolated for qRT-PCR analysis, as described below. To collect the individual hearts, the transgenic zebrafish strain $\mathrm{Tg}$ (myl7: EGFP) was used. The homozygous mutants were separated from their siblings according to their mobility and pectoral fin phenotype. The embryos were disrupted by pipetting up and down several times (depending on the developmental stages) with a $10 \mu \mathrm{L}$ pipette. Next, the individual hearts were picked up by pipettes under a fluorescence stereomicroscope. The individual hearts were pooled into the same tube, and the total RNA was extracted using TransZol Up Plus RNA kit (Transgen, China). The cDNA was reverse-transcribed from the total RNA and used for qRT-PCR analysis.

\subsection{Calcium Signaling Detection Using Fluo-4 AM}

The calcium signal was detected according to the kit manual (Molecular Probes). Briefly, the hearts isolated from the $48 \mathrm{hpf} \mathrm{rnf}^{-/-}$and wild-type embryos were transferred to a $20 \mathrm{~mm}$ glass-bottom cell culture dish containing external control solution (ECS, con- 
taining $140 \mathrm{mM} \mathrm{NaCl}, 4 \mathrm{mM} \mathrm{KCl}, 1.8 \mathrm{mM} \mathrm{CaCl}_{2}, 1 \mathrm{mM} \mathrm{MgCl} 2,10 \mathrm{mM}$ glucose, and $10 \mathrm{mM}$ HEPES (pH 7.4)). Next, the embryos were incubated in an ECS medium containing $5 \mu \mathrm{M}$ Fluo-4 AM, $10 \mu \mathrm{M}$ blebbistatin, and $0.35 \mathrm{mM}$ probenecid (Molecular Probes) for $30 \mathrm{~min}$. The blebbistatin was added to uncouple the excitation-contraction process in the zebrafish embryonic hearts [62]. After being rinsed twice with ECS containing $0.35 \mathrm{mM}$ probenecid, the hearts were transferred into ECS containing $0.35 \mathrm{mM}$ probenecid. Images were taken with a confocal laser scanning microscope (Leica SP8 DLS).

\subsection{Imaging, Quantification and Statistical Analysis}

The area of pericardial edema of $72 \mathrm{hpf}$ embryos was measured by marking the edema region on photos using ImageJ. The heart rates were counted manually under a dissecting microscope (WPI). The heart beating frequency in one minute per embryo was calculated manually. For the transmission electron microscopy of the cardiac muscle structure, the 80 hpf $r n f 2^{-/-}$and wild-type embryos were fixed, embedded in the Spur resin, and then sectioned. The sections were stained with uranyl acetate and lead citrate. The images were taken with a Hitachi TEM system (Japan). The data were analyzed with the GraphPad Prism 7.0 software. The values are presented as mean \pm SEM. The $p$-values were calculated using two-tailed Student's test, ${ }^{*} p \leq 0.05,{ }^{* *} p \leq 0.01,{ }^{* * *} p \leq 0.001$.

Supplementary Materials: The following are available online at https:/ /www.mdpi.com/article/10 $.3390 /$ ijms222111368/s1.

Author Contributions: Conceptualization, Y.S.; formal analysis, X.P.; funding acquisition, Y.S.; investigation, X.P. and Y.Z.; resources, X.P. and G.F.; visualization, X.P.; writing-original draft, X.P. and Y.S.; writing-review and editing, X.P. and Y.S. All authors have read and agreed to the published version of the manuscript.

Funding: This research was funded by Strategic Priority Research Program of Chinese Academy of Sciences (grant number XDB31000000) and National Natural Science Foundation of China (grant number 31671526).

Institutional Review Board Statement: The study was conducted according to the guidelines of the Care and Use of Laboratory Animals, and approved by the Ethics Committee of Institute of Hydrobiology, Chinese Academy of Sciences, Wuhan, China.

Acknowledgments: We thank all the researchers in our laboratory. We are grateful for the help from Fang Zhou at the analysis and testing center of Institute of Hydrobiology, Chinese Academy of Sciences in confocal imaging as well as Xingxing Wu and Shaoting Jia at Institute of Hydrobiology, Chinese Academy of Sciences in histological analysis.

Conflicts of Interest: The authors declare no conflict of interest.

\section{References}

1. Bakkers, J.; Verhoeven, M.C.; Abdelilah-Seyfried, S. Shaping the zebrafish heart: From left-right axis specification to epithelial tissue morphogenesis. Dev. Biol. 2009, 330, 213-220. [CrossRef]

2. Houk, A.R.; Yelon, D. Strategies for analyzing cardiac phenotypes in the zebrafish embryo. In Zebrafish: Cellular and Developmental Biology, Pt B: Developmental Biology; Detrich, H.W., Westerfield, M., Zon, L.I., Eds.; Elsevier: Amsterdam, The Netherlands, 2016; pp. 335-368.

3. Miura, G.I.; Yelon, D. A guide to analysis of cardiac phenotypes in the zebrafish embryo. Methods Cell Biol. 2011, 101, 161-180. [PubMed]

4. Peterson, R.T.; Mably, J.D.; Chen, J.-N.; Fishman, M.C. Convergence of distinct pathways to heart patterning revealed by the small molecule concentramide and the mutation heart-and-soul. Curr. Biol. 2001, 11, 1481-1491. [CrossRef]

5. Yelon, D.; Horne, S.A.; Stainier, D.Y.R. Restricted expression of cardiac myosin genes reveals regulated aspects of heart tube assembly in zebrafish. Dev. Biol. 1999, 214, 23-37. [CrossRef] [PubMed]

6. Yang, J.; Shih, Y.-H.; Xu, X. Understanding cardiac sarcomere assembly with Zebrafish genetics. Anat. Rec. Adv. Integr. Anat. Evol. Biol. 2014, 297, 1681-1693. [CrossRef] [PubMed]

7. Gregorio, C.C.; Antin, P.B. To the heart of myofibril assembly. Trends Cell Biol. 2000, 10, 355-362. [CrossRef]

8. Squire, J.M. Architecture and function in the muscle sarcomere. Curr. Opin. Struct. Biol. 1997, 7, 247-257. [CrossRef]

9. Wilczewski, C.M.; Hepperla, A.J.; Shimbo, T.; Wasson, L.; Robbe, Z.; Davis, I.; Wade, P.; Conlon, F.L. CHD4 and the NuRD complex directly control cardiac sarcomere formation. Proc. Natl. Acad. Sci. USA 2018, 115, 6727-6732. [CrossRef] 
10. Van Weerd, J.H.; Christoffels, V.M. The formation and function of the cardiac conduction system. Development 2016, 143, 197-210. [CrossRef]

11. Peal, D.S.; Lynch, S.N.; Milan, D.J. Patterning and development of the atrioventricular canal in zebrafish. J. Cardiovasc. Transl. Res. 2011, 4, 720-726. [CrossRef]

12. Walsh, E.C.; Stainier, D.Y.R. UDP-glucose dehydrogenase required for cardiac valve formation in zebrafish. Science 2001, $293,1670$. [CrossRef]

13. Chi, N.C.; Shaw, R.; De Val, S.; Kang, G.; Jan, L.; Black, B.; Stainier, D.Y. Foxn4 directly regulates tbx2b expression and atrioventricular canal formation. Genes Dev. 2008, 22, 734-739. [CrossRef]

14. Verhoeven, M.C.; Haase, C.; Christoffels, V.M.; Weidinger, G.; Bakkers, J. Wnt signaling regulates atrioventricular canal formation upstream of BMP and Tbx2. Birth Defects Res. Part A Clin. Mol. Teratol. 2011, 91, 435-440. [CrossRef]

15. Puskaric, S.; Schmitteckert, S.; Mori, A.D.; Glaser, A.; Schneider, K.U.; Bruneau, B.; Blaschke, R.J.; Steinbeisser, H.; Rappold, G. Shox 2 mediates Tbx5 activity by regulating Bmp4 in the pacemaker region of the developing heart. Hum. Mol. Genet. 2010, 19, 4625-4633. [CrossRef]

16. Hashem, S.I.; Lam, M.L.; Mihardja, S.S.; White, S.M.; Lee, R.J.; Claycomb, W.C. Shox2 regulates the pacemaker gene program in embryoid bodies. Stem Cells Dev. 2013, 22, 2915-2926. [CrossRef]

17. Di Croce, L.; Helin, K. Transcriptional regulation by Polycomb group proteins. Nat. Struct. Mol. Biol. 2013, 20, 1147-1155. [CrossRef]

18. Hamish, W.K.; Nadezda, A.F.; Neil, P.B.; Robert, J.K. PRC1 shapes the nucleosome landscape but not accessibility at target genes Genome Res. 2018, 2018, 14.

19. Brockdorff, N. Polycomb complexes in X chromosome inactivation. Philos. Trans. R. Soc. B Biol. Sci. 2017, $372,20170021$. [CrossRef] [PubMed]

20. Almeida, M.; Pintacuda, G.; Masui, O.; Koseki, Y.; Gdula, M.; Cerase, A.; Brown, D.; Mould, A.; Innocent, C.; Nakayama, M.; et al. PCGF3/5-PRC1 initiates Polycomb recruitment in X chromosome inactivation. Science 2017, 356, 1081-1084. [CrossRef] [PubMed]

21. Zhao, W.; Tong, H.; Huang, Y.; Yan, Y.; Teng, H.; Xia, Y.; Jiang, Q.; Qin, J. Essential role for Polycomb group protein Pcgf6 in Embryonic stem cell maintenance and a noncanonical Polycomb Repressive Complex 1 (PRC1) integrity. J. Biol. Chem. 2017, 292, 2773-2784. [CrossRef] [PubMed]

22. Dupret, B.; Völkel, P.; Le Bourhis, X.; Angrand, P.-O. The Polycomb group protein Pcgf1 is dispensable in Zebrafish but involved in early growth and aging. PLoS ONE 2016, 11, e0158700. [CrossRef]

23. Chan, H.L.; Beckedorff, F.; Zhang, Y.; Garcia-Huidobro, J.; Jiang, H.; Colaprico, A.; Bilbao, D.; Figueroa, M.E.; LaCava, J.; Shiekhattar, R.; et al. Polycomb complexes associate with enhancers and promote oncogenic transcriptional programs in cancer through multiple mechanisms. Nat. Commun. 2018, 9, 3377. [CrossRef]

24. Banito, A.; Li, X.; Laporte, A.N.; Roe, J.-S.; Sanchez-Vega, F.; Huang, C.-H.; Dancsok, A.R.; Hatzi, K.; Chen, C.-C.; Tschaharganeh, D.F.; et al. The SS18-SSX oncoprotein hijacks KDM2B-PRC1.1 to drive synovial sarcoma. Cancer Cell 2018, 33, 527-541.e8. [CrossRef] [PubMed]

25. Schuettengruber, B.; Bourbon, H.-M.; Di Croce, L.; Cavalli, G. Genome regulation by Polycomb and trithorax: 70 years and counting. Cell 2017, 171, 34-57. [CrossRef] [PubMed]

26. De Napoles, M.; Mermoud, J.E.; Wakao, R.; Tang, Y.A.; Endoh, M.; Appanah, R.; Brockdorff, N.; Silva, J.; Otte, A.P.; Vidal, M.; et al. Polycomb group proteins Ring1A/B link ubiquitylation of histone H2A to heritable gene silencing and X inactivation. Dev. Cell 2004, 7, 663-676. [CrossRef] [PubMed]

27. Tavares, L.; Dimitrova, E.; Oxley, D.; Webster, J.; Poot, R.; Demmers, J.; Bezstarosti, K.; Taylor, S.; Ura, H.; Koide, H.; et al. RYBP-PRC1 complexes mediate H2A ubiquitylation at polycomb target sites independently of PRC2 and H3K27me3. Cell 2012, 148, 664-678. [CrossRef] [PubMed]

28. Gao, Z.; Zhang, J.; Bonasio, R.; Strino, F.; Sawai, A.; Parisi, F.; Kluger, Y.; Reinberg, D. PCGF homologs, CBX proteins, and RYBP define functionally distinct PRC1 family complexes. Mol. Cell 2012, 45, 344-356.e8. [CrossRef]

29. Van der Velden, Y.U.; Wang, L.; van Lohuizen, M.; Haramis, A.-P.G. The Polycomb group protein Ring1b is essential for pectoral fin development. Development 2012, 139, 2210-2220. [CrossRef]

30. Van der Velden, Y.U.; Wang, L.; Cano, L.Q.; Haramis, A.-P.G. The Polycomb group protein Ring1b/Rnf2 is specifically required for craniofacial development. PLoS ONE 2013, 8, e73997. [CrossRef]

31. Chrispijn, N.D.; Elurbe, D.M.; Mickoleit, M.; Aben, M.; De Bakker, D.E.; Andralojc, K.M.; Huisken, J.; Bakkers, J.; Kamminga, L.M. Loss of the Polycomb group protein Rnf2 results in derepression of tbx-transcription factors and defects in embryonic and cardiac development. Sci. Rep. 2019, 9, 4327. [CrossRef]

32. Bakkers, J. Zebrafish as a model to study cardiac development and human cardiac disease. Cardiovasc. Res. 2011, 91, 279-288. [CrossRef]

33. Keegan, B.R.; Meyer, D.; Yelon, D. Organization of cardiac chamber progenitors in the zebrafish blastula. Development 2004, 131, 3081-3091. [CrossRef]

34. Stainier, D.Y. Zebrafish genetics and vertebrate heart formation. Nat. Rev. Genet. 2001, 2, 39. [CrossRef] [PubMed]

35. Puceat, M. Embryological origin of the endocardium and derived valve progenitor cells: From developmental biology to stem cell-based valve repair. Biochim. Et Biophys. Acta Mol. Cell Res. 2013, 1833, 917-922. [CrossRef] [PubMed] 
36. He, A.; Ma, Q.; Cao, J.; Von Gise, A.; Zhou, P.; Xie, H.; Zhang, B.; Hsing, M.; Christodoulou, D.C.; Cahan, P.; et al. Polycomb Repressive Complex 2 regulates normal development of the mouse heart. Circ. Res. 2012, 110, 406-415. [CrossRef] [PubMed]

37. San, B.; Chrispijn, N.; Wittkopp, N.; van Heeringen, S.; Lagendijk, A.K.; Aben, M.; Bakkers, J.; Ketting, R.; Kamminga, L.M. Normal formation of a vertebrate body plan and loss of tissue maintenance in the absence of ezh2. Sci. Rep. 2016, 6, 24658. [CrossRef] [PubMed]

38. Shirai, M.; Takihara, Y.; Morisaki, T. Pcgf5 contributes to PRC1 (Polycomb Repressive Complex 1) in developing cardiac cells. In Etiology and Morphogenesis of Congenital Heart Disease: From Gene Function and Cellular Interaction to Morphology; Nakanishi, T., Ed.; Springer: Tokyo, Japan, 2016; pp. 305-312.

39. Dickinson, M.E.; Flenniken, A.M.; Ji, X.; Teboul, L.; Wong, M.D.; White, J.K.; Meehan, T.F.; Weninger, W.J.; Westerberg, H.; Adissu, H.; et al. High-throughput discovery of novel developmental phenotypes. Nature 2016, 537, 508-514. [CrossRef]

40. Piunti, A.; Shilatifard, A. The roles of Polycomb repressive complexes in mammalian development and cancer. Nat. Rev. Mol. Cell Biol. 2021, 22, 326-345. [CrossRef]

41. Osugi, T.; Shirai, M.; Koga, H.; Nishiguchi, S.; Yamauchi-Takihara, K.; Takihara, Y. Rae28, a member of the mammalian Polycomb group of genes, is required for maintenance of NKX2.5 expression in cardiac development. Circulation 2000, $102,99$.

42. Voncken, J.W.; Roelen, B.A.; Roefs, M.; de Vries, S.; Verhoeven, E.; Marino, S.; Deschamps, J.; van Lohuizen, M. Rnf2 (Ring1b) deficiency causes gastrulation arrest and cell cycle inhibition. Proc. Natl. Acad. Sci. USA 2003, 100, 2468-2473. [CrossRef]

43. Buchwald, G.; Van Der Stoop, P.; Weichenrieder, O.; Perrakis, A.; Van Lohuizen, M.; Sixma, T.K. Structure and E3-ligase activity of the Ring-Ring complex of polycomb proteins Bmi1 and Ring1b. EMBO J. 2006, 25, 2465-2474. [CrossRef]

44. Illingworth, R.S.; Moffat, M.; Mann, A.R.; Read, D.; Hunter, C.J.; Pradeepa, M.M.; Adams, I.R.; Bickmore, W.A. The E3 ubiquitin ligase activity of RING1B is not essential for early mouse development. Genes Dev. 2015, 29, 1897-1902. [CrossRef] [PubMed]

45. Eskeland, R.; Leeb, M.; Grimes, G.R.; Kress, C.; Boyle, S.; Sproul, D.; Gilbert, N.; Fan, Y.; Skoultchi, A.I.; Wutz, A.; et al. Ring1B compacts chromatin structure and represses gene expression independent of histone ubiquitination. Mol. Cell 2010, 38, $452-464$. [CrossRef] [PubMed]

46. Cao, R.; Wang, L.; Wang, H.; Xia, L.; Erdjument-Bromage, H.; Tempst, P.; Jones, R.S.; Zhang, Y. Role of histone H3 lysine 27 methylation in polycomb-group silencing. Science 2002, 298, 1039-1043. [CrossRef] [PubMed]

47. Boyle, S.; Flyamer, I.M.; Williamson, I.; Sengupta, D.; Bickmore, W.A.; Illingworth, R.S. A central role for canonical PRC1 in shaping the 3D nuclear landscape. Genes Dev. 2020, 34, 931-949. [CrossRef] [PubMed]

48. Clark, K.A.; McElhinny, A.S.; Beckerle, M.C.; Gregorio, C.C. Striated muscle cytoarchitecture: An intricate web of form and function. Annu. Rev. Cell Dev. Biol. 2002, 18, 637-706. [CrossRef]

49. Pierrat, O.A.; Paudyal, A.; Woodruff, J.; Koroleva, O.; Boateng, S.Y. The exon junction complex senses energetic stress and regulates contractility and cell architecture in cardiac myocytes. Biosci. Rep. 2017, 37, BSR20170707. [CrossRef]

50. Delgado-Olguín, P.; Huang, Y.; Li, X.; Christodoulou, D.; Seidman, C.E.; Seidman, J.G.; Tarakhovsky, A.; Bruneau, B.G. Epigenetic repression of cardiac progenitor gene expression by Ezh2 is required for postnatal cardiac homeostasis. Nat. Genet. 2012, 44, 343-347. [CrossRef]

51. Min, J.R.; Zhang, Y.; Xu, R.M. Structural basis for specific binding of polycomb chromodomain to histone H3 methylated at Lys 27. Genes Dev. 2003, 17, 1823-1828. [CrossRef]

52. Wang, H.; Wang, L.; Erdjument-Bromage, H.; Vidal, M.; Tempst, P.; Jones, R.S.; Zhang, Y. Role of histone H2A ubiquitination in Polycomb silencing. Nature 2004, 431, 873-878. [CrossRef]

53. Blackledge, N.P.; Farcas, A.M.; Kondo, T.; King, H.W.; McGouran, J.F.; Hanssen, L.L.; Ito, S.; Cooper, S.; Kondo, K.; Koseki, Y.; et al. Variant PRC1 complex-dependent H2A ubiquitylation drives PRC2 recruitment and polycomb domain formation. Cell 2014, 157, 1445-1459. [CrossRef] [PubMed]

54. Sugishita, H.; Kondo, T.; Ito, S.; Nakayama, M.; Yakushiji-Kaminatsui, N.; Kawakami, E.; Koseki, Y.; Ohinata, Y.; Sharif, J.; Harachi, M.; et al. Variant PCGF1-PRC1 links PRC2 recruitment with differentiation-associated transcriptional inactivation at target genes. Nat. Commun. 2021, 12, 5341. [CrossRef] [PubMed]

55. Wolf, C.M.; Berul, C.I. Inherited conduction system abnormalities-One group of diseases, many genes. J. Cardiovasc. Electrophysiol. 2006, 17, 446-455. [CrossRef]

56. Jensen, B.; Boukens, B.J.; Postma, A.; Gunst, Q.D.; Hoff, M.J.B.V.D.; Moorman, A.F.M.; Wang, T.; Christoffels, V.M. Identifying the evolutionary building blocks of the cardiac conduction system. PLoS ONE 2012, 7, e44231. [CrossRef] [PubMed]

57. Bakker, M.L.; Boukens, B.J.; Mommersteeg, M.T.; Brons, J.F.; Wakker, V.; Moorman, A.F.; Christoffels, V.M. Transcription factor Tbx3 is required for the specification of the atrioventricular conduction system. Circ. Res. 2008, 102, 1340-1349. [CrossRef]

58. Chi, N.C.; Shaw, R.; Jungblut, B.; Huisken, J.; Ferrer, T.; Arnaout, R.; Scott, I.; Beis, D.; Xiao, T.; Baier, H.; et al. Genetic and physiologic dissection of the vertebrate cardiac conduction system. PLoS Biol. 2008, 6, e109. [CrossRef] [PubMed]

59. Westerfield, M. The Zebrafish Book: A Guide for the Laboratory Use of Zebrafish (Danio Rerio); University of Oregon Press: Eugene, OR, USA, 1995.

60. Sun, X.; Chen, J.; Zhang, Y.; Munisha, M.; Dougan, S.; Sun, Y. Mga modulates bmpr1a activity by antagonizing Bs69 in Zebrafish. Front. Cell Dev. Biol. 2018, 6, 126. [CrossRef] 
61. Thisse, C.; Thisse, B. High-resolution in situ hybridization to whole-mount zebrafish embryos. Nat. Protoc. 2008, 3, 59-69. [CrossRef] [PubMed]

62. Jou, C.J.; Spitzer, K.W.; Tristani-Firouzi, M. Blebbistatin effectively uncouples the excitation-contraction process in Zebrafish embryonic heart. Cell. Physiol. Biochem. 2010, 25, 419-424. [CrossRef] 